\title{
Hemizygous loss of NF2 detected by fluorescence in situ hybridization is useful for the diagnosis of malignant pleural mesothelioma
}

\author{
Yoshiaki Kinoshita $\mathbb{( i )}^{1,2} \cdot$ Makoto Hamasaki $^{1} \cdot$ Masayo Yoshimura $\mathbb{C}^{1} \cdot$ Shinji Matsumoto $^{1} \cdot$ Akinori Iwasaki $^{3} \cdot$ \\ Kazuki Nabeshima ${ }^{1}$
}

Received: 4 April 2019 / Revised: 27 May 2019 / Accepted: 27 May 2019 / Published online: 23 June 2019

(c) The Author(s), under exclusive licence to United States \& Canadian Academy of Pathology 2019

\begin{abstract}
Neurofibromatosis type 2 (NF2) gene, a tumor suppressor gene located on chromosome 22q12.2, is frequently abnormal in mesothelioma. Recent studies have revealed the effectiveness of diagnostic assays for differentiating malignant pleural mesothelioma from reactive mesothelial hyperplasia. These include detection of homozygous deletion of the $9 \mathrm{p} 21$ locus by fluorescence in situ hybridization (FISH) (9p21 FISH), loss of expression of BAP1 as detected by immunohistochemistry, and loss of expression of methylthioadenosine phosphorylase (MTAP) as detected by immunohistochemistry. However, the application of FISH detection of NF2 gene deletion (NF2 FISH) in differentiation of malignant pleural mesothelioma from reactive mesothelial hyperplasia has not been fully evaluated. In this study, we investigated whether NF2 FISH, either alone or in a combination with other diagnostic assays (9p21 FISH, MTAP immunohistochemistry, and BAP1 immunohistochemistry), is effective for distinguishing malignant pleural mesothelioma from reactive mesothelial hyperplasia. This study cohort included malignant pleural mesothelioma $(n=47)$ and reactive mesothelial hyperplasia cases $(n=27)$ from a period between 2001 and 2017. We used FISH to examine deletion status of NF2 and 9p21 and immunohistochemistry to examine expression of MTAP and BAP1 in malignant pleural mesothelioma and in reactive mesothelial hyperplasia. Hemizygous NF2 loss (chromosome 22 monosomy or hemizygous deletion) was detected in 25 of 47 (53.2\%) mesothelioma cases. None of the mesothelioma cases showed homozygous NF2 deletion. Hemizygous NF2 loss showed 53.2\% sensitivity and $100 \%$ specificity in differentiating malignant pleural mesothelioma from reactive mesothelial hyperplasia. A combination of NF2 FISH, 9p21 FISH, and BAP1 immunohistochemistry yielded greater sensitivity (100\%) than that detected for either diagnostic assay alone $(53.2 \%$ for $N F 2$ FISH, $78.7 \%$ for $9 \mathrm{p} 21$ FISH, $70.2 \%$ for MTAP immunohistochemistry, or $57.4 \%$ for BAP1 immunohistochemistry). Thus, NF2 FISH in combination with other diagnostic assays is effective for distinguishing malignant pleural mesothelioma from reactive mesothelial hyperplasia.
\end{abstract}

Note: A portion of this work was presented in March 2019 at the 108th annual meeting of the United States and Canadian Academy of Pathology in National Harbor, Maryland, USA.

Kazuki Nabeshima

kaznabes@fukuoka-u.ac.jp

1 Department of Pathology, Fukuoka University Hospital and School of Medicine, Fukuoka, Japan

2 Department of Respiratory Medicine, Fukuoka University Hospital and School of Medicine, Fukuoka, Japan

3 Department of Thoracic Surgery, Fukuoka University Hospital and School of Medicine, Fukuoka, Japan

\section{Introduction}

Malignant pleural mesothelioma is the most common primary neoplasm of the pleura [1]. Its incidence is closely linked with exposure to asbestos fibers [1]. The prognosis of malignant pleural mesothelioma is generally poor with a median survival period of 9.2 to 14 months [2, 3]. Although histologic diagnosis of malignant pleural mesothelioma is not always straightforward [1], diagnosis at an early stage may lead to a better prognosis $[4,5]$.

Mesothelioma has frequent genetic alterations in Cyclindependent kinase inhibitor $2 A(C D K N 2 A) / p 16$ found in the 9p21 locus (35-62\%), BRCA1-associated protein 1 (BAP1) (21-63\%), and neurofibromatosis type 2 (NF2) (14-50\%) $[6,7]$. Recent studies have revealed the usefulness of assays 
detecting changes in the 9p21 locus and BAP1 expression for mesothelioma diagnosis-homozygous deletion of the 9p21 locus as detected by fluorescence in situ hybridization (FISH) (9p21 FISH) [8-17] and expression loss of BAP1 as detected by immunohistochemistry [12, 15-21]. In addition, we have previously reported that immunohistochemical detection of methylthioadenosine phosphorylase (MTAP) is a reliable surrogate assay for 9p21 FISH [14, 15, 17, 22-24]. These aforementioned assays can provide a definitive diagnosis of mesothelioma and can facilitate differentiation of malignant mesothelial proliferations from benign proliferations with $100 \%$ specificity $[1,15-17,23,25]$. Although the sensitivity of either of these assays on their own is insufficient, the combined use of 9p21 FISH (or MTAP immunohistochemistry) and BAP1 immunohistochemistry enhances the sensitivity [12, 14-17, 21, 23, 25].

The NF2 is a tumor suppressor gene which is located on chromosome 22q12.2 and encodes moesin-ezrin-radixin-like protein (merlin) [26]. This protein modulates signal transduction pathways including Hippo pathways and mammalian target of rapamycin (mTOR) pathways, which regulate cell proliferation, growth, and apoptosis [26]. Inactivation of the $N F 2$ gene has been observed in certain benign and malignant tumors including neurofibromatosis type 2, sporadic schwannoma, meningioma, and mesothelioma [26].

Genetic mutations in NF2 are frequently observed in mesothelioma [6, 7, 27]. Singhi et al. [28] showed the prevalence of $N F 2$ hemizygosity in $35 \%$ of peritoneal mesotheliomas cases using FISH. However, the application of NF2 FISH for differentiating malignant pleural mesothelioma from reactive mesothelial hyperplasia has not been established in pleural mesotheliomas. In this study, we investigated whether NF2 FISH, either alone or in a combination with other diagnostic assays, is effective for distinguishing malignant pleural mesothelioma from reactive mesothelial hyperplasia.

\section{Materials and methods}

\section{Case selection}

This study included malignant pleural mesothelioma and reactive mesothelial hyperplasia cases identified from the pleural lesion files of the Department of Pathology, Fukuoka University Hospital, which includes pleural diseases such as malignant pleural mesothelioma, reactive mesothelial hyperplasia, various pleuritis, metastatic carcinomas, and primary thoracic sarcomas, between the years of 2001 and 2017. Histological diagnosis and classification of mesothelioma were performed according to the 2015 World Health Organization guidelines [29]. The mesothelial nature of each tumor was confirmed using immunohistochemical assays. Calretinin, podoplanin (D2-40), and Wilms' tumor-1 (WT-1) were used as positive mesothelial markers, while thyroid transcription factor-1 (TTF-1), Ber-EP4, claudin-4, and carcinoembryonic antigen (CEA) were used as negative markers.

Each mesothelioma case was classified into three histologic types (epithelioid, biphasic, or sarcomatoid). Classification of either epithelioid or sarcomatoid mesothelioma required at least $90 \%$ of the tumor to be composed of this morphologic pattern [1, 29]. Biphasic mesothelioma classification required both components to represent at least $10 \%$ of the tumor $[1,29]$.

Anonymous use of redundant tissues is part of the standard treatment agreement with patients at our hospital, provided no objection is expressed. The Fukuoka University Hospital Institutional Review Board approved the study protocol (approval number: 11-7-11).

\section{FISH assay}

A representative tissue specimen from each case was selected for FISH analysis. The FISH studies were carried out on 4- $\mu$ mthick tissue sections as previously described [11, 13, 15, 23]. Sections were deparaffinized and rehydrated using descending alcohol dilutions, washed with $2 \mathrm{x}$ saline-sodium citrate (SSC), exposed to pretreatment solution at $80^{\circ} \mathrm{C}$ for $30 \mathrm{~min}$ (PathVysion HER2 DNA probe kit; Vysis, Downers Grove, IL, USA), and digested with pepsin solution (Sigma-Aldrich, Tokyo, Japan) at $37^{\circ} \mathrm{C}$ for $90 \mathrm{~min}$. After refixation in $10 \%$ buffered formalin at room temperature (RT) for $10 \mathrm{~min}$, the sections were treated with 2x SSC, dehydrated in ethanol, dried, and exposed to Vysis LSI p16/CEP 9 probe (Abbott Japan, Tokyo, Japan) and NF2/CEN 22q probe (Abnova, Walnut, CA, USA). Tissue sections were denatured at $80^{\circ} \mathrm{C}$ for $10 \mathrm{~min}$ in the probe solution provided (Abbott Japan), followed by hybridization at $37^{\circ} \mathrm{C}$ for $20 \mathrm{~h}$ in ThermoBrite (Abbott Japan). Finally, the tissue sections were washed with $2 \times$ SSC containing $0.3 \%$ Tween 20 (Sigma, St. Louis, MO, USA) at $72{ }^{\circ} \mathrm{C}$ for $2 \mathrm{~min}$ and then with $2 \times$ SSC containing $0.1 \%$ Tween 20 at RT for $5 \mathrm{~min}$. Nuclei were counterstained with 4', 6-diamidino-2-phenylindole (DAPI) in Antifade reagent (Vector Laboratories, Burlingame, CA, USA). Analyses were performed using a fluorescence microscope (Axio Imager Z1; Carl Zeiss MicroImaging, Jena, Germany) and Isis analysis system (MetaSystems, Altlussheim, Germany) equipped with filter sets with single and dual-band exciters for spectrum green, spectrum orange, and DAPI (UV $360 \mathrm{~nm}$ ).

Lymphocytes in each preparation served as internal controls and showed two signals per FISH probe. The use of internal controls helped to confirm that loss of FISH signals was not due to preanalytical factors such as fixation or processing. We evaluated the FISH signal patterns in at least 100 mesothelial cells per sample. A cutoff value of $10 \%$ was set for homozygous deletion of 9p21 FISH as described previously [11, 14-16, 30]. 
Fig. 1 Hematoxylin-eosin staining and NF2 dual-color fluorescence in situ hybridization in reactive mesothelial hyperplasia (a, b) and malignant pleural mesothelioma (c, d). a, b mesothelial cells with a normal copy number showing 2 spectrum red and 2 spectrum green signals. c, $\mathbf{d}$ mesothelioma cells with hemizygous NF2 loss showing 1 spectrum red and 1 spectrum green signals (pattern of chromosome 22 monosomy)
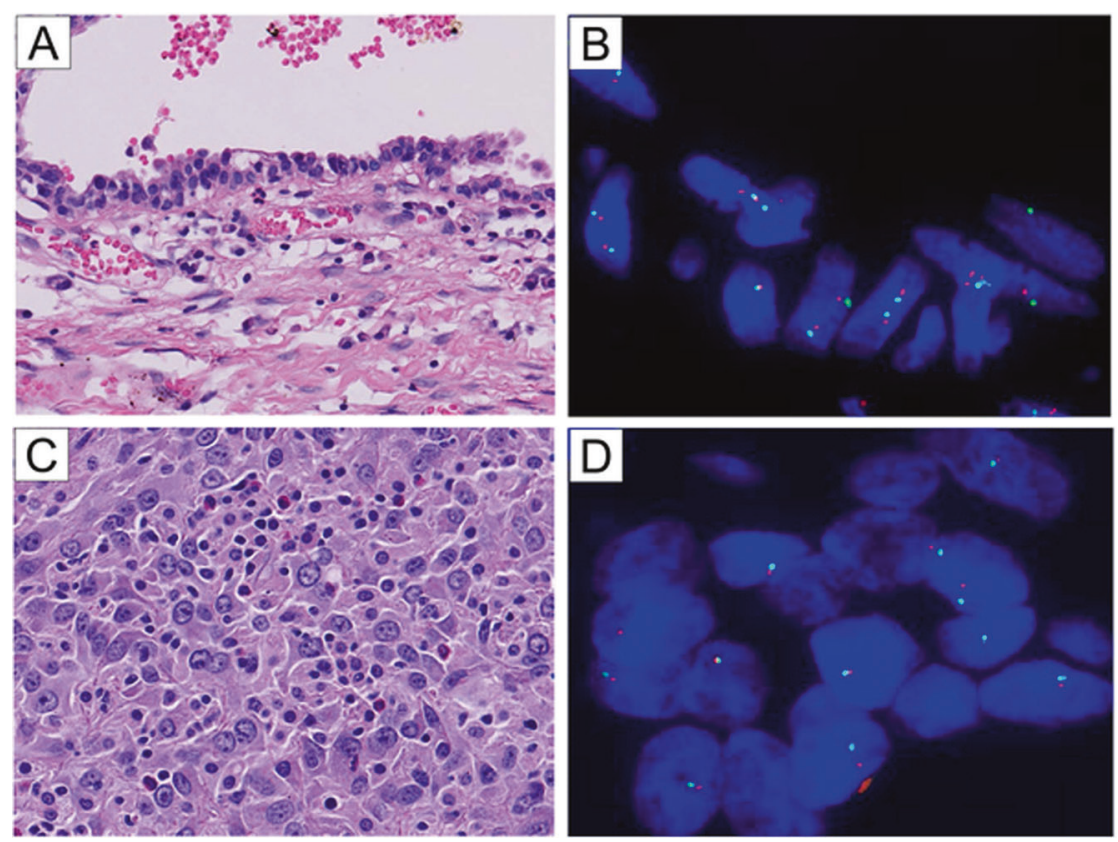

As for NF2 FISH, signal cutoff values for each deletion pattern [hemizygous deletion, chromosome 22 monosomy, homozygous deletion, and one fluorescein isothiocyanate (FITC) signal were established according to the mean frequency $(\%)+3$ standard deviations (SDs) for each of the patterns in the reactive mesothelial hyperplasia cases [11]. We defined one Spectrum Red (SpR) and two Spectrum Green (SpG) signals (1SpR/2SpG) as hemizygous deletion, $1 \mathrm{SpR} / 1 \mathrm{SpG}$ as chromosome 22 monosomy, $0 \mathrm{SpR} / 2 \mathrm{SpG}$ as homozygous deletion, and $0 \mathrm{SpR} / 1 \mathrm{SpG}$ as one FITC signal. In this study, hemizygous NF2 loss indicates hemizygous $N F 2$ deletion $(1 \mathrm{SpR} / 2 \mathrm{SpG})$ or chromosome 22 monosomy (1SpR/1SpG), as described previously [28].

\section{Immunohistochemistry of MTAP and BAP1}

A representative tissue specimen from each case was selected for immunohistochemical analysis. Immunostaining was performed on 4 - $\mu$ m-thick sections which were mounted on a glass microscope slide. After blocking the endogenous peroxidase activity using blocking reagent (included in the Dako Envision Kit, Dako, California, USA) for $5 \mathrm{~min}$ at RT, epitopes were retrieved using $\mathrm{pH} 9.0$ TrisEDTA buffer for $40 \mathrm{~min}$ at $95^{\circ} \mathrm{C}$ followed by incubation with mouse monoclonal antibody MTAP clone 2G4 (Abnova, Taipei, Taiwan; 1:100 dilution; RT $30 \mathrm{~min}$ ) or mouse monoclonal anti-human BAP-1 clone C-4 (Santa Cruz Biotechnology, Santa Cruz, CA, USA; 1:50 dilution; RT $45 \mathrm{~min}$ ). The sections were then washed and incubated with ChemMate EnVision kit (Dako). Immunoreacted cells were visualized using diaminobenzidine (Dako, California, USA) followed by a hematoxylin counterstain.
Non-mesothelial cells that were immunoreactive to BAP1 and MTAP (e.g., inflammatory cells including histiocytes and lymphocytes, fibroblasts, pneumocytes, and endothelial cells) served as internal positive controls in each staining protocol. BAP1 immunohistochemistry revealed staining in the nucleus, and BAP1 loss in tumor cells was defined as complete nuclear loss [14, 15, 23]. Cytoplasmic staining was interpreted as a nonspecific reaction. MTAP immunohistochemistry revealed cytoplasmic as well as nuclear staining, and MTAP loss as detected by immunohistochemistry in tumor cells was defined as cytoplasmic staining at an intensity lower than the internal positive control $[14,15,23,31]$. We set the cutoff value at $50 \%$ for MTAP immunohistochemistry and BAP1 immunohistochemistry, as described previously [14, 15, 19, 23].

\section{Statistical analysis}

Continuous data are shown as the group means $( \pm \mathrm{SD})$, and categorical data are shown as a percentage of the group. Fisher's exact test was used to compare categorical variables. The differences in the mean values of continuous data were assessed using Student's $t$-test for unpaired data. We performed survival analysis using overall survival as the endpoint, beginning at the time of resection. The survival curves were plotted via the Kaplan-Meier method, and the differences between survival curves were analyzed using the log-rank test. A $p$-value $<0.05$ was considered to indicate statistical significance. All statistical analyses were performed using $\mathrm{R}$ (version 3.2.2; R Foundation for Statistical Computing, Vienna, Austria). 
Fig. 2 Hematoxylin-eosin staining and 9p21 dual-color fluorescence in situ hybridization in reactive mesothelial hyperplasia $(\mathbf{a}, \mathbf{b})$ and malignant pleural mesothelioma (c, d). a, b mesothelial cells with a normal copy number showing 2 spectrum red and 2 spectrum green signals. c, $\mathbf{d}$ mesothelioma cells with homozygous $9 \mathrm{p} 21$ deletion showing the pattern of 0 spectrum red and 2 spectrum green signals
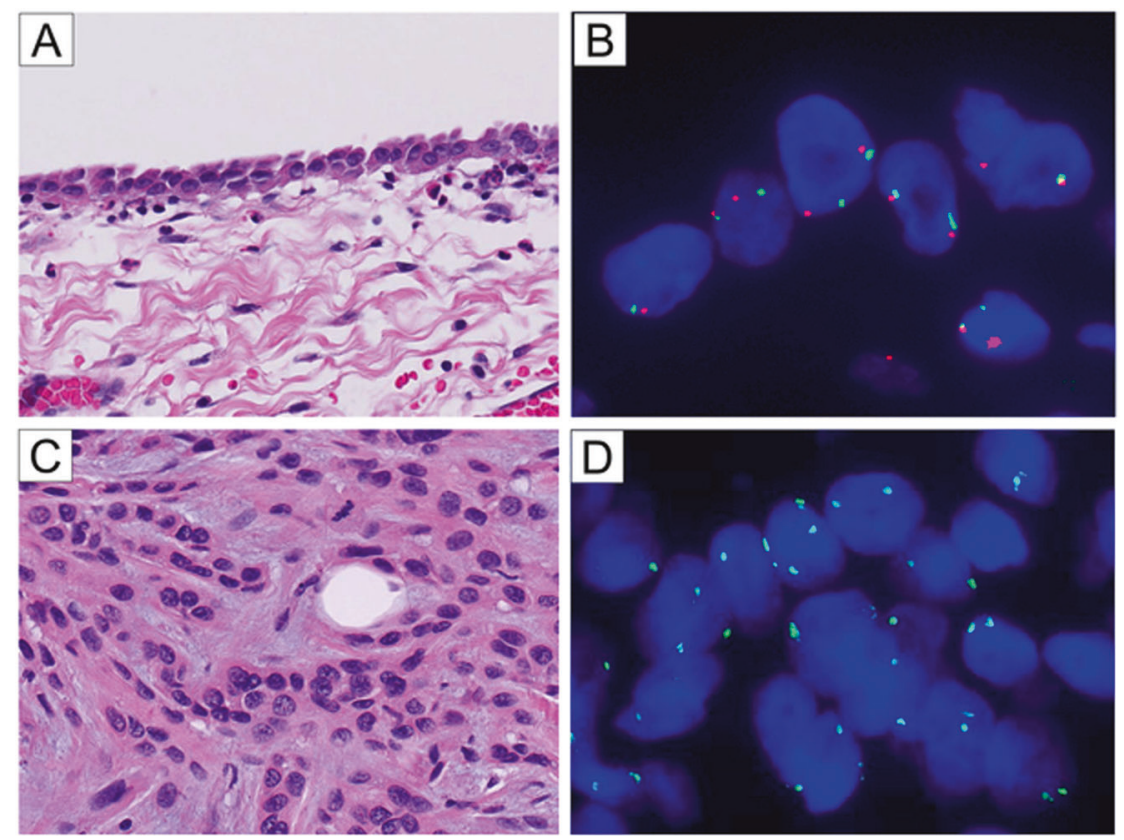

\section{Results}

\section{Clinicopathological characteristics}

Forty-seven cases with malignant pleural mesothelioma and 27 cases with reactive mesothelial hyperplasia were included in this study. Mesothelioma cases comprised 41 males and 6 females with a mean age of 67.3 years (range: $45-86$ years) and included 32 epithelioid mesothelioma, 5 sarcomatoid mesothelioma, and 10 biphasic mesothelioma cases. Reactive mesothelial hyperplasia cases included 26 males and 1 female with a mean age of 33.0 years (range: $18-78$ years).

\section{NF2 and 9p21 FISH}

Mesothelial cells with a normal copy number for $9 \mathrm{p} 21$ or $N F 2$ have two red signals ( $9 \mathrm{p} 21$ probe or $N F 2$ probe) and two green signals (chromosome 9 or chromosome 22 centromeric probes). In mesothelioma, $N F 2$ deletion as detected by FISH was characterized by hemizygous loss (hemizygous deletion: $1 \mathrm{SpR} / 2 \mathrm{SpG}$ or chromosome 22 monosomy: $1 \mathrm{SpR} / 1 \mathrm{SpG}$ ). Mesothelioma cells with homozygous $9 \mathrm{p} 21$ deletion showed $0 \mathrm{SpR} / 2 \mathrm{SpG}$ signal profile. Representative examples of NF2 FISH and 9p21 FISH in reactive mesothelial hyperplasia and malignant pleural mesothelioma cases are shown in Figs. 1 and 2, respectively.

\section{Determination of the cutoff values for NF2 FISH}

The signal cutoff values (calculated as indicated in the Materials and Methods section) for NF2 FISH were
Table 1 The cutoff values for NF2 FISH

\begin{tabular}{ll}
\hline$N F 2$ FISH signal & $\begin{array}{l}\text { Cutoff values (mean }+3 \mathrm{SD} \\
\text { in RMH) }(\%)\end{array}$ \\
\hline Hemizygous loss & \\
Hemizygous deletion (1SpR/2SpG) & 18.2 \\
Chromosome 22 monosomy (1SpR/ & 48.4 \\
1SpG) & \\
Homozygous deletion (0SpR/2SpG) & 5.5 \\
One FITC signal (0SpR/1SpG) & 12.9 \\
\hline
\end{tabular}

FISH fluorescence in situ hybridization, $S D$ standard deviation, $R M H$ reactive mesothelial hyperplasia, $S p R$ spectrum red, $S p G$ Spectrum Green, FITC fluorescein isothiocyanate

determined to be $18.2 \%$ for hemizygous deletion ( $1 \mathrm{SpR} /$ $2 \mathrm{SpG}), 48.4 \%$ for chromosome 22 monosomy (1SpR/ $1 \mathrm{SpG}), 5.5 \%$. for homozygous deletion $(0 \mathrm{SpR} / 2 \mathrm{SpG})$, and $12.9 \%$ for one FITC signal $(0 \mathrm{SpR} / 1 \mathrm{SpG})($ Table 1$)$.

\section{MTAP and BAP1 immunostaining}

BAP1 immunohistochemistry shows staining in the nucleus. BAP1 loss in mesothelioma showed nuclear staining at an intensity lower than that in the internal positive controls. MTAP immunohistochemistry showed cytoplasmic staining as well as nuclear staining and MTAP loss in mesothelioma showed cytoplasmic staining at an intensity lower than that in the internal positive controls. BAP1 loss was detected in 27 of the 47 mesothelioma cases $(57.4 \%)$, whereas none of the reactive mesothelial hyperplasia cases showed BAP1 loss. Meanwhile, MTAP loss was detected in 33 of the 47 
Fig. 3 Representative examples of MTAP and BAP1 immunohistochemistry in malignant pleural mesothelioma. Non-mesothelial cells that were reactive to BAP1 and MTAP served as internal positive controls in each staining protocol. Preserved BAP1 immunohistochemistry (nuclear staining) (a), loss of BAP1 immunohistochemistry (b), preserved MTAP immunohistochemistry (cytoplasmic staining) (c), and loss of MTAP immunohistochemistry (d) are shown
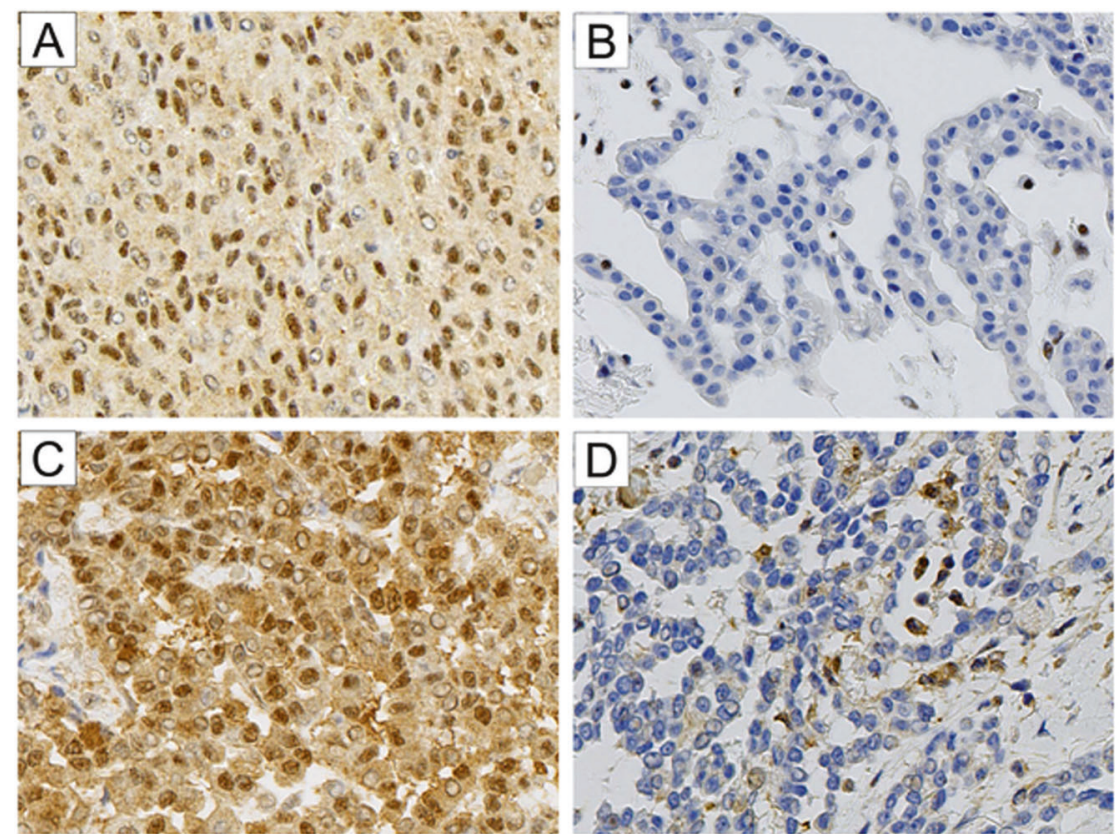

mesothelioma cases (70.2\%), while none of the reactive mesothelial hyperplasia cases showed MTAP loss. Representative examples of MTAP and BAP1 immunostaining in mesothelioma cases are shown in Fig. 3.

\section{Deletion status of $\mathbf{N F 2}$ using FISH}

Hemizygous NF2 loss was detected in 25 of the 47 mesothelioma cases $(53.2 \%$; $4.3 \%$ for $1 \mathrm{SpR} / 2 \mathrm{SpG}$ and $48.9 \%$ for $1 \mathrm{SpR} / 1 \mathrm{SpG})$, while homozygous NF2 deletion was not detected in mesothelioma cases. Neither hemizygous loss nor homozygous deletion of $N F 2$ was observed in reactive mesothelial hyperplasia cases.

The correlation between hemizygous NF2 loss and other parameters in malignant pleural mesothelioma is summarized in Table 2. Hemizygous NF2 loss was not associated with age $(p=0.39)$, gender $(p=0.67)$, or histologic subtype $(p=0.32)$, statistically. It is noteworthy that hemizygous NF2 loss was also not associated with homozygous 9p21 deletion $(p=0.73)$, MTAP loss as detected by immunohistochemistry $(p=$ 0.52 ), or BAP1 loss as detected by immunohistochemistry $(p=0.77)$, statistically.

\section{Sensitivity and specificity of detection assays for discriminating malignant pleural mesothelioma from reactive mesothelial hyperplasia}

The results for each detection assay in the 47 mesothelioma cases are shown in Fig. 4. The sensitivity and specificity of each detection assay for discriminating malignant pleural
Table 2 The correlation between hemizygous NF2 loss and other parameters in malignant pleural mesothelioma

\begin{tabular}{llccc}
\hline Factor & Group & $\begin{array}{c}\text { Hemizygous } \\
\text { loss }(n=25)\end{array}$ & $\begin{array}{c}\text { Wild type } \\
(n=22)\end{array}$ & $p$-value \\
\hline Age & $\geq 70$ & $9(36.0)$ & $11(50.0)$ & 0.39 \\
& $<70$ & $16(64.0)$ & $11(50.0)$ & \\
Gender & Female & $4(16.0)$ & $2(9.1)$ & 0.67 \\
& Male & $21(84.0)$ & $20(90.9)$ & \\
Histologic & Epithelioid & $18(72.0)$ & $14(63.6)$ & 0.32 \\
subtype & Sarcomatoid & $1(4.0)$ & $4(18.2)$ & \\
& Biphasic & $6(24.0)$ & $4(18.2)$ & \\
9p21 FISH & Homozygous & $19(76.0)$ & $18(81.8)$ & 0.73 \\
& deletion & & & \\
& Wild type & $6(24.0)$ & $4(18.2)$ & \\
MTAP IHC & Loss & $16(68.0)$ & $17(77.3)$ & 0.52 \\
& Retained & $8(32.0)$ & $5(22.7)$ & \\
BAP1 IHC & Loss & $15(60.0)$ & $12(54.5)$ & 0.77 \\
& Retained & $10(40.0)$ & $10(45.5)$ & \\
\hline
\end{tabular}

FISH fluorescence in situ hybridization, $I H C$ immunohistochemistry

mesothelioma from reactive mesothelial hyperplasia are summarized in Table 3. None of the reactive mesothelial hyperplasia cases showed loss of expression of BAP1 or MTAP as detected by immunohistochemistry or deletion of NF2 or $9 \mathrm{p} 21$ as detected by FISH. Thus, each detection assay was characterized by a $100 \%$ specificity. Assay sensitivities were $53.2 \%$ for NF2 FISH, $78.7 \%$ for 9p21 FISH, $70.2 \%$ for MTAP immunohistochemistry, and $57.4 \%$ for BAP1 immunohistochemistry. The assay sensitivity was increased to $93.6 \%$ for the combination of 9p21 FISH and 

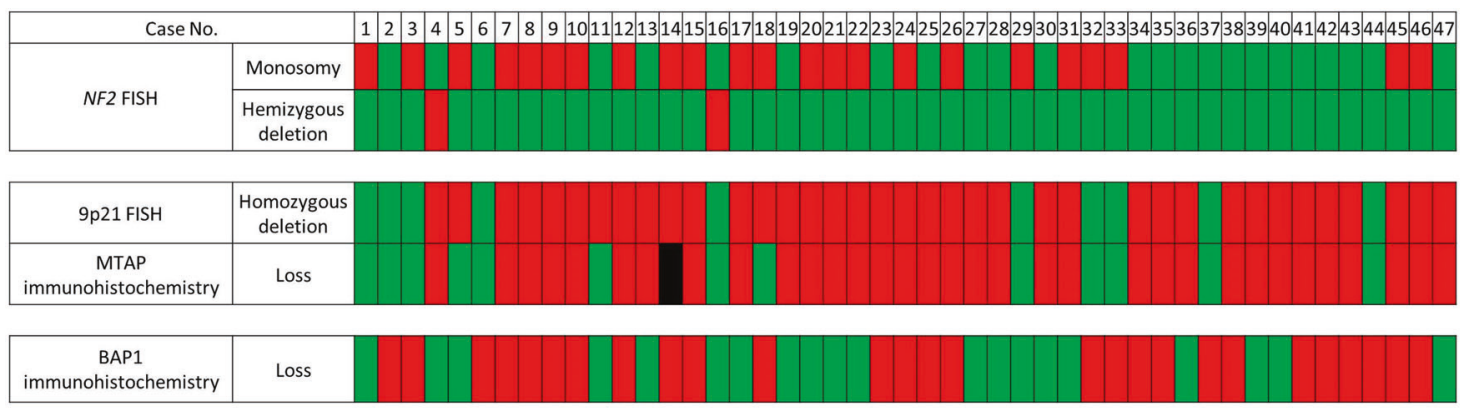

Loss or deleted

Fig. 4 The results of each detection assay in the 47 cases of malignant pleural mesothelioma. Green represent retained expression as determined by immunohistochemistry or fluorescence in situ hybridization (FISH); red represents loss or deleted expression as detected by immunohistochemistry or FISH; black represents undetermined expression as detected by immunohistochemistry. Note: For 9p21 FISH, deleted indicates homozygous deletion; for NF2 FISH, deleted indicates hemizygous loss
Table 3 Sensitivity and specificity of each detection assay for discriminating malignant pleural mesothelioma from reactive mesothelial hyperplasia

\begin{tabular}{|c|c|c|c|c|c|c|}
\hline & \multicolumn{2}{|c|}{$\operatorname{MPM}(n=47)$} & \multicolumn{2}{|c|}{$\mathrm{RMH}(n=27)$} & \multirow[t]{2}{*}{ Sensitivity (\%) } & \multirow[t]{2}{*}{ Specificity (\%) } \\
\hline & $\begin{array}{l}\text { Loss or } \\
\text { deleted }^{\mathrm{b}}\end{array}$ & Retained & $\begin{array}{l}\text { Loss or } \\
\text { deleted }^{\mathrm{b}}\end{array}$ & Retained & & \\
\hline NF2 FISH & 25 & 22 & 0 & 27 & 53.2 & 100 \\
\hline 9p21 FISH & 37 & 10 & 0 & 27 & 78.7 & 100 \\
\hline MTAP IHC & 33 & 13 & 0 & 27 & 70.2 & 100 \\
\hline BAP1 IHC & 27 & 20 & 0 & 27 & 57.4 & 100 \\
\hline BAP1 IHC/9p21 FISH & 44 & 3 & 0 & 27 & 93.6 & 100 \\
\hline BAP1/MTAP IHC & 42 & 5 & 0 & 27 & 89.4 & 100 \\
\hline $\begin{array}{l}\text { NF2 FISH/ BAP1 } \\
\text { IHC/9p21 FISH }\end{array}$ & 47 & 0 & 0 & 27 & 100 & 100 \\
\hline $\begin{array}{l}N F 2 \text { FISH/ BAP1 } \\
\text { IHC/MTAP IHC }\end{array}$ & 46 & 1 & 0 & 27 & 97.9 & 100 \\
\hline
\end{tabular}

$M P M$ malignant pleural mesothelioma, $R M H$ reactive mesothelial hyperplasia, FISH fluorescence in situ hybridization, $I H C$ immunohistochemistry

${ }^{a}$ One case was excluded from sensitivity and specificity calculation for MTAP IHC due to data interpretation issues

${ }^{\mathrm{b}}$ For 9p21 FISH, deleted indicates homozygous deletion; for NF2 FISH, deleted indicates hemizygous loss
BAP1 immunohistochemistry and to $89.4 \%$ for the combination of MTAP immunohistochemistry and BAP1 immunohistochemistry. A triple combination of NF2 FISH, 9p21 FISH, and BAP1 immunohistochemistry yielded greater sensitivity $(100 \%)$ than that detected for either diagnostic assay alone or for the combination of either two of these assays.

\section{Survival analysis}

Overall survival curves for patients with malignant pleural mesothelioma are shown in Fig. 5. As expected, there was a significant difference in overall survival based on the histologic type $(p=0.001)$ (Fig. 5a). Similarly, 9p21 homozygous deletion detected by FISH and MTAP loss detected by immunohistochemistry were clearly associated with a shorter overall survival $(p<0.001$ and $p<0.001$, respectively) (Fig. 5b, c). However, BAP1 loss detected by immunohistochemistry or hemizygous NF2 loss detected by FISH were not associated with a shorter overall survival (Fig. 5d, e).

\section{Discussion}

In this study, we revealed that NF2 loss as detected by FISH was characterized by hemizygous loss. Hemizygous $N F 2$ loss was not associated with FISH detection of homozygous 9p21 deletion, immunohistochemical detection of MTAP loss, or immunohistochemical detection of BAP1 loss. 

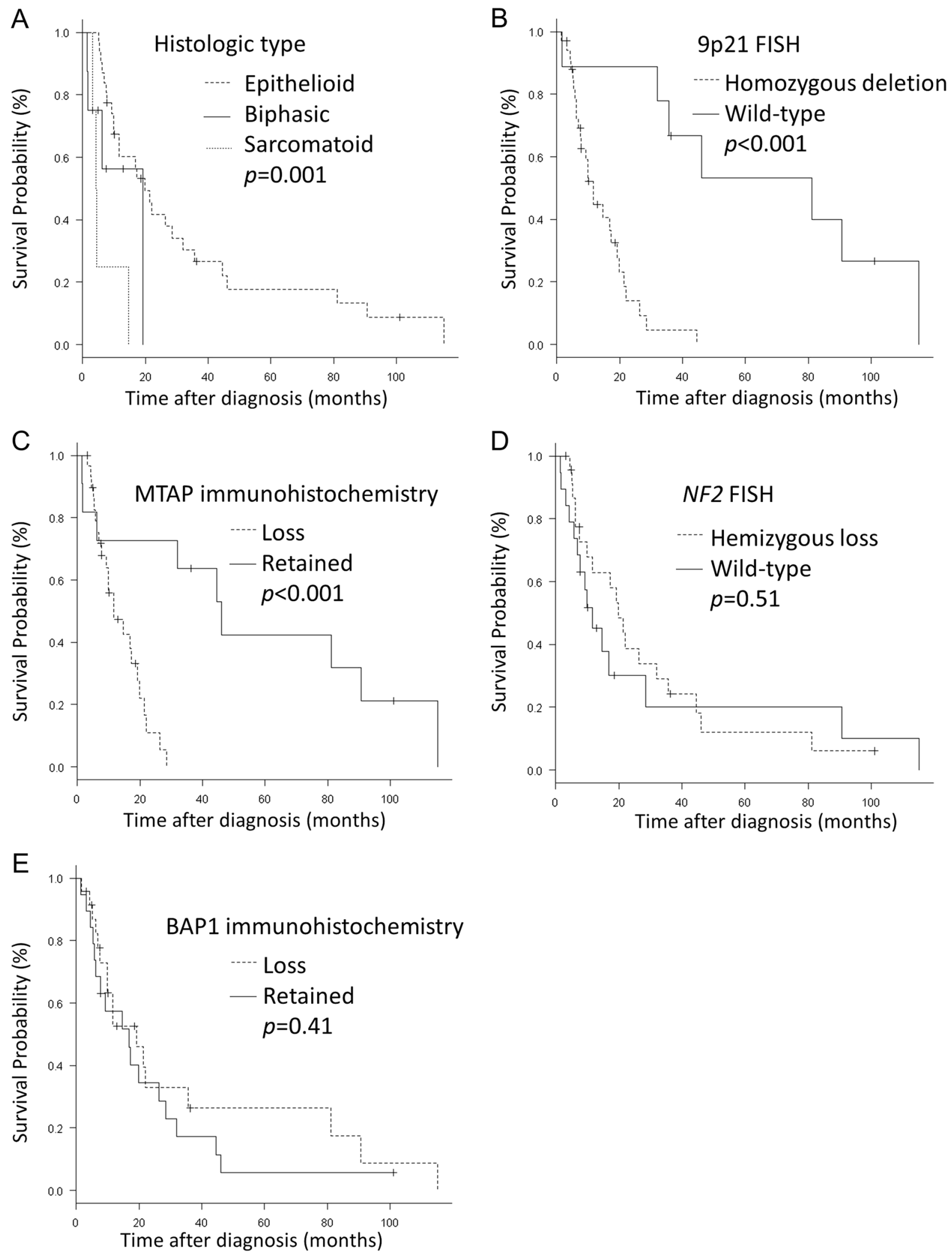

Fig. 5 Overall survival curves for patients with malignant pleural mesothelioma. The effect of histologic type (a), the status of 9p21 FISH (b), MTAP immunohistochemistry (c), NF2 FISH (d), and BAP1 immunohistochemistry (e) on overall survival curves is shown 
Hemizygous NF2 loss showed $53.2 \%$ sensitivity and $100 \%$ specificity in differentiating malignant pleural mesothelioma from reactive mesothelial hyperplasia. In this cohort, $N F 2$ FISH in a combination with diagnostic assays for $9 \mathrm{p} 21$ homozygous deletion (FISH) and BAP1 expression loss (immunohistochemistry) is effective for distinguishing malignant pleural mesothelioma from reactive mesothelial hyperplasia at $100 \%$ specificity and sensitivity.

Karyotypic studies have previously shown that clonal abnormalities in chromosome 22 are the most frequent site of chromosomal aberrations in mesothelioma (39.2-65.0\%) $[32,33]$. A limited number of studies have investigated the frequency of $N F 2$ deletion in mesothelioma using FISH $[28,34,35]$. Vivero et al. [34] reported that the frequency of $N F 2$ deletion detected by FISH was $72.5 \%$ (37/51 cases) in malignant pleural mesothelioma (cutoff values: $4 \%$ for monosomy and $8 \%$ for hemizygous deletion). Takeda et al. [35]. reported that the frequency of chromosomal loss at 22q12 detected by FISH was 38\% in malignant pleural mesothelioma cases (cutoff values: not described). Singhi et al. [28] showed that NF2 loss as detected by FISH was identified in $35 \%$ of peritoneal mesotheliomas (cutoff values: not described). The frequency of NF2 deletion detected by FISH is highly influenced by the cutoff value used for the analysis of FISH signal. Using procedure described in Materials and Methods section, the cutoff values calculated in our study were $48.4 \%$ for monosomy and $18.2 \%$ for hemizygous deletion. Applying these criteria, hemizygous NF2 loss detected by FISH could differentiate malignant pleural mesothelioma from reactive mesothelial hyperplasia with $53.2 \%$ sensitivity and $100 \%$ specificity.

In the above-mentioned peritoneal mesothelioma study, $N F 2$ loss as detected by FISH was characterized by hemizygous loss (35\%), and chromosome 22 monosomy (30\%) was more frequent than hemizygous $N F 2$ deletion (5\%) [28]. In our study, the frequency of hemizygous NF2 loss in malignant pleural mesothelioma (53.2\%) was higher than that in peritoneal mesothelioma. Meanwhile, similar to the peritoneal mesothelioma study, chromosome 22 monosomy (48.9\%) was more frequent than hemizygous NF2 deletion (4.3\%). In contrast to 9p21 FISH, homozygous NF2 deletion was not detected in any of the mesothelioma cases in our study as well as in other previous reports [17, 28]. Thus, NF2 loss as detected by FISH appears to be characterized by hemizygous loss in mesothelioma with chromosome 22 monosomy being more frequent than hemizygous $N F 2$ deletion.

Clinically, 9p21 FISH and BAP1 immunohistochemistry have $100 \%$ specificity for differentiating malignant mesothelial proliferations from benign proliferations [1, 1417, 23, 25]. Chiosea et al. [9] first reported the usefulness of 9p21 FISH for detecting mesothelioma cells in tissue sections. For differentiating malignant pleural mesothelioma from reactive mesothelial hyperplasia, 9p21 FISH shows a sensitivity of $45-85 \%$ in epithelioid mesothelioma [8-12, 14, 17] and 67-100\% in sarcomatoid mesothelioma $[21,23]$. The role of BAP1 in mesothelioma was first suggested in reports of BAP1 somatic mutations by Bott et al. [27] and of germline mutations in BAP1 by Testa et al. [36]. For differentiation between malignant pleural mesothelioma from reactive mesothelial hyperplasia, BAP1 immunohistochemistry shows a sensitivity of $56-81 \%$ in epithelioid mesothelioma $[12,17,18]$ and $0-63 \%$ in sarcomatoid mesothelioma [12, 18, 21, 23]. In addition, we have previously reported that MTAP immunohistochemistry is an acceptable surrogate assay for 9p21 FISH, a finding that was also supported by Berg et al. $[14,15,17,23]$. In our previous study, $65-75 \%$ of the malignant pleural mesothelioma cases harboring 9p21 homozygous deletion (detected by FISH), demonstrated MTAP loss detected by immunohistochemistry [14, 15, 23]. In addition, MTAP immunohistochemistry distinguished malignant mesothelial proliferations from benign proliferations with a specificity of $100 \%$ and a sensitivity of $45.1 \%$ in epithelioid mesothelioma and $80 \%$ in sarcomatoid mesothelioma [14, 15, 23].

In the current study, hemizygous NF2 loss as detected by FISH was not correlated with FISH detection of 9p21 homozygous deletion, immunohistochemical detection of MTAP loss, or immunohistochemical detection of BAP1 loss. Therefore, the combined use of NF2 FISH and these three diagnostic assays can enhance the sensitivity of malignant pleural mesothelioma differentiation from reactive mesothelial hyperplasia. Indeed, a triple combination of NF2 FISH, 9p21 FISH, and BAP1 immunohistochemistry yielded greater sensitivity $(100 \%)$ than that detected for either diagnostic assay alone or either combination of two of these assays.

Technical challenges and cost can limit FISH assays from widespread adoption. Unfortunately, immunohistochemical assays for the NF2 gene have not shown efficacy in the differentiation of malignant pleural mesothelioma from reactive mesothelial hyperplasia, to date. In their study examining the usefulness of merlin immunohistochemistry for the differentiation of mesothelioma from a benign proliferation, Sheffield et al. [37] concluded that merlin immunohistochemistry is unsuitable due to its low sensitivity (4\%) and the discordance between NF2 genetic changes and immunohistochemical detection of merlin expression. Recent studies have identified alterations in the components of the Hippo signaling cascade in mesothelioma cells, including large tumor suppressor homolog 2 (LATS2) and their downstream effectors Yes-associated protein (YAP) and transcriptional coactivator with the PDZ-binding motif (TAZ) complex [37, 38]. The application of immunohistochemical detection of LATS and YAP/TAZ immunohistochemistry for the differentiation of 
mesothelioma from a benign proliferation was also investigated; however, these immunohistochemical assays were also found to be unsuitable due to their low sensitivity or specificity [37]. Nevertheless, further investigation may be needed to fully elucidate whether immunohistochemical detection of NF2 has a role in mesothelioma diagnosis.

In mesothelioma, functional inactivation of $N F 2 /$ merlin is known to be associated with mesothelial oncogenesis, invasiveness, spreading, and migration [39, 40]. A recent study showed that low merlin expression as detected by immunohistochemistry is related to the poor prognosis of malignant pleural mesothelioma [41]. In peritoneal mesothelioma, Singhi et al. [28] showed that hemizygous NF2 deletion as detected by FISH was significantly associated with a poor prognosis. However, in our current study, there was no significant prognostic implications of hemizygous NF2 loss in malignant pleural mesothelioma. Merlin is inactivated by not only genetic alteration but also by epigenetic events (e.g., phosphorylation) [39]. Thurneysen et al. [39] revealed that merlin is rendered inactive upon phosphorylation in all malignant pleural mesothelioma cases wherein no NF2 truncation could be detected. The lack of association between hemizygous $N F 2$ loss and survival observed in our study might be because merlin is frequently inactivated even in mesothelioma cases harboring wild-type $N F 2$ gene.

Our study has a few limitations. First, this study is a single-center retrospective study, and the number of patients is relatively small due to the rarity of the disease. Validation studies are preferred to confirm these results. Second, several old samples were utilized in our study with some cases dating back to 2001. However, for FISH analysis, we obtained two red and green FISH signals in the internal control cells as described in the "Materials and methods" section; this validated the reactivity of FISH analysis. Third, inactivation of $N F 2$ gene in the remaining allele of chromosome 22 showing hemizygous loss could not be examined. This is relevant in light of the fact that inactivation of NF2 gene occurs via a two-hit mechanism [40, 42]. However, previous studies have shown that more than half of mesothelioma cases harboring allelic loss of $22 \mathrm{q}$ also showed NF2 gene mutation in the remaining allele [43, 44]. More studies are warranted to confirm these findings.

In conclusion, FISH assay shows that the most common molecular alteration of $N F 2$ in malignant pleural mesothelioma is hemizygous loss. NF2 FISH in a combination with other diagnostic assays was effective for distinguishing malignant pleural mesothelioma from reactive mesothelial hyperplasia.

Acknowledgements The authors thank Ms. K. Yano and Ms. M. Onitsuka, Department of Pathology, Fukuoka University School of Medicine and Hospital, for their skilled assistance with the 9p21 FISH and immunohistochemical assays. This work was supported in part by Grants-in-Aid for Scientific Research (\#24590495) from the Japanese
Society for the Promotion of Science; grants from the Research Center for Advanced Molecular Medicine, Fukuoka University; funds (\#171032) from the Central Research Institute of Fukuoka University; and grant from the Clinical Research Promotion Foundation.

\section{Compliance with ethical standards}

Conflict of interest The authors declare that they have no conflict of interest.

Publisher's note: Springer Nature remains neutral with regard to jurisdictional claims in published maps and institutional affiliations.

\section{References}

1. Husain AN, Colby TV, Ordóñez NG, Allen TC, Attanoos RL, Beasley $\mathrm{MB}$, et al. Guidelines for pathologic diagnosis of malignant mesothelioma 2017 update of the consensus statement from the International Mesothelioma Interest Group. Arch Pathol Lab Med. 2018;142:89-108.

2. Yates DH, Corrin B, Stidolph PN, Browne K. Malignant mesothelioma in south east England: clinicopathological experience of 272 cases. Thorax. 1997;52:507-12.

3. Beckett P, Edwards J, Fennell D, Hubbard R, Woolhouse I, Peake MD. Demographics, management and survival of patients with malignant pleural mesothelioma in the National Lung Cancer Audit in England and Wales. Lung Cancer. 2015;88:344-8.

4. Rusch VW, Rosenzweig K, Venkatraman E, Leon L, Raben A, Harrison L, et al. A phase II trial of surgical resection and adjuvant highdose hemithoracic radiation for malignant pleural mesothelioma. J Thorac Cardiovasc Surg. 2001;122:788-95.

5. Sugarbaker DJ, Jaklitsch MT, Bueno R, Richards W, Lukanich J, Mentzer SJ, et al. Prevention, early detection, and management of complications after 328 consecutive extrapleural pneumonectomies. J Thorac Cardiovasc Surg. 2004;128:138-46.

6. Kato S, Tomson BN, Buys TPH, Elkin SK, Carter JL, Kurzrock R. Genomic landscape of malignant mesotheliomas. Mol Cancer Ther. 2016;15:2498-507.

7. Guo G, Chmielecki J, Goparaju C, Heguy A, Dolgalev I, Carbone $\mathrm{M}$, et al. Whole-exome sequencing reveals frequent genetic alterations in BAP1, NF2, CDKN2A, and CUL1 in malignant pleural mesothelioma. Cancer Res. 2015;75:264-9.

8. Illei PB, Ladanyi M, Rusch VW, Zakowski MF. The use of CDKN2A deletion as a diagnostic marker for malignant mesothelioma in body cavity effusions. Cancer. 2003;99:51-6.

9. Chiosea S, Krasinskas A, Cagle PT, Mitchell KA, Zander DS, Dacic S. Diagnostic importance of 9p21 homozygous deletion in malignant mesotheliomas. Mod Pathol. 2008;21:742-7.

10. Chung CT-S, Santos GDC, Hwang DM, Ludkovski O, Pintilie M, Squire JA, et al. FISH assay development for the detection of p16/ CDKN2A deletion in malignant pleural mesothelioma. J Clin Pathol. 2010;63:630-4.

11. Matsumoto S, Nabeshima K, Kamei T, Hiroshima K, Kawahara $\mathrm{K}$, Hata $\mathrm{S}$, et al. Morphology of 9p21 homozygous deletionpositive pleural mesothelioma cells analyzed using fluorescence in situ hybridization and virtual microscope system in effusion cytology. Cancer Cytopathol. 2013;121:415-22.

12. Sheffield BS, Hwang HC, Lee AF, Thompson K, Rodriguez S, Tse $\mathrm{CH}$, et al. BAP1 immunohistochemistry and p16 FISH to separate benign from malignant mesothelial proliferations. Am J Surg Pathol. 2015;39:977-82.

13. Hamasaki M, Matsumoto S, Abe S, Hamatake D, Kamei T, Hiroshima $\mathrm{K}$, et al. Low homozygous/high heterozygous deletion 
status by p16 FISH correlates with a better prognostic group than high homozygous deletion status in malignant pleural mesothelioma. Lung Cancer. 2016;99:155-61.

14. Hida T, Hamasaki M, Matsumoto S, Sato A, Tsujimura T, Kawahara K, et al. Immunohistochemical detection of MTAP and BAP1 protein loss for mesothelioma diagnosis: comparison with 9p21 FISH and BAP1 immunohistochemistry. Lung Cancer. 2017;104:98-105.

15. Kinoshita Y, Hida T, Hamasaki M, Matsumoto S, Sato A, Tsujimura $\mathrm{T}$, et al. A combination of MTAP and BAP1 immunohistochemistry in pleural effusion cytology for the diagnosis of mesothelioma. Cancer Cytopathol. 2018;126:54-63.

16. Hida $T$, Hamasaki $M$, Matsumoto $S$, Sato A, Tsujimura $T$, Kawahara K, et al. BAP1 immunohistochemistry and p16 FISH results in combination provide higher confidence in malignant pleural mesothelioma diagnosis: ROC analysis of the two tests. Pathol Int. 2016;66:563-70.

17. Berg KB, Dacic S, Miller C, Cheung S, Churg A. Utility of methylthioadenosine phosphorylase compared with BAP1 immunohistochemistry, and CDKN2A and NF2 fluorescence in situ hybridization in separating reactive mesothelial proliferations from epithelioid malignant mesotheliomas. Arch Pathol Lab Med. 2018;137:1255-61.

18. Nasu M, Emi M, Pastorino S, Tanji M, Powers A, Luk H, et al. High incidence of somatic BAP1 alterations in sporadic malignant mesothelioma. J Thorac Oncol. 2015;10:565-76.

19. Yoshimura M, Kinoshita Y, Hamasaki M, Matsumoto S, Hida T, Oda Y, et al. Diagnostic application of BAP1 immunohistochemistry to differentiate pleural mesothelioma from metastatic pleural tumours. Histopathology. 2017;71:1011-4.

20. Carbone M, Shimizu D, Napolitano A, Tanji M, Pass HI, Yang $\mathrm{H}$, et al. Positive nuclear BAP1 immunostaining helps differentiate non-small cell lung carcinomas from malignant mesothelioma. Oncotarget. 2016;7:59314-21.

21. Hwang HC, Pyott S, Rodriguez S, Cindric A, Carr A, Michelsen $\mathrm{C}$, et al. BAP1 immunohistochemistry and p16 FISH in the diagnosis of sarcomatous and desmoplastic mesotheliomas. Am J Surg Pathol. 2016;40:714-8.

22. Churg A, Nabeshima K, Ali G, Bruno R, Fernandez-Cuesta L, Galateau-Salle F. Highlights of the 14th international mesothelioma interest group meeting: Pathologic separation of benign from malignant mesothelial proliferations and histologic/molecular analysis of malignant mesothelioma subtypes. Lung Cancer. 2018;124:95-101.

23. Kinoshita Y, Hamasaki M, Yoshimura M, Matsumoto S, Sato A, Tsujimura T, et al. A combination of MTAP and BAP1 immunohistochemistry is effective for distinguishing sarcomatoid mesothelioma from fibrous pleuritis. Lung Cancer. 2018; 125:198-204

24. Yoshimura M, Kinoshita Y, Hamasaki M, Matsumoto S, Hida $\mathrm{T}$, Oda $\mathrm{Y}$, et al. Highly expressed EZH2 in combination with BAP1 and MTAP loss, as detected by immunohistochemistry, is useful for differentiating malignant pleural mesothelioma from reactive mesothelial hyperplasia. Lung Cancer. 2019; 130:187-93.

25. Churg A, Sheffield BS, Galateau-Salle F. New markers for separating benign from malignant mesothelial proliferations: are we there yet? Arch Pathol Lab Med. 2016;140:318-21.

26. Sekido Y. Genomic abnormalities and signal transduction dysregulation in malignant mesothelioma cells. Cancer Sci. 2010; 101:1-6.

27. Bott M, Brevet M, Taylor BS, Shimizu S, Ito T, Wang L, et al. The nuclear deubiquitinase BAP1 is commonly inactivated by somatic mutations and 3p21.1 losses in malignant pleural mesothelioma. Nat Genet. 2011;43:668-72.
28. Singhi AD, Krasinskas AM, Choudry HA, Bartlett DL, Pingpank JF, Zeh HJ, et al. The prognostic significance of BAP1, NF2, and CDKN2A in malignant peritoneal mesothelioma. Mod Pathol. 2016;29:14-24.

29. Galateau-Salle F, Churg A, Roggli V, Chirieac LR, Attanoos R, Borczuk A, et al. Mesothelial tumours. In: Travis W, Brambilla E, Burke A, Marx A, Nicholson A, editors. WHO Classification of Tumours of the Lung, Pleura, Thymus and Heart. 4th edn, Lyon, France: International Agency for Research on Cancer Press; 2015. pp. 156-75.

30. Hida T, Matsumoto S, Hamasaki M, Kawahara K, Tsujimura T, Hiroshima K, et al. Deletion status of p16 in effusion smear preparation correlates with that of underlying malignant pleural mesothelioma tissue. Cancer Sci. 2015;106:1635-41.

31. Hamasaki M, Kinoshita Y, Yoshimura M, Matsumoto S, Kamei $\mathrm{T}$, Hiroshima $\mathrm{K}$, et al. Cytoplasmic MTAP expression loss detected by immunohistochemistry correlates with $9 \mathrm{p} 21$ homozygous deletion detected by FISH in pleural effusion cytology of mesothelioma. Histopathology. https://doi.org/10.1111/his.13872.

32. Lee WC, Testa JR. Somatic genetic alterations in human malignant mesothelioma (review). Int J Oncol. 1999;14:181-8.

33. Flejter WL, Li FP, Antman KH, Testa JR. Recurring loss involving chromosomes 1,3 , and 22 in malignant mesothelioma: possible sites of tumor suppressor genes. Genes Chromosomes Cancer. 1989;1:148-54.

34. Vivero M, Bueno R, Chirieac LR. Clinicopathologic and genetic characteristics of young patients with pleural diffuse malignant mesothelioma. Mod Pathol. 2018;31:122-31.

35. Takeda M, Kasai T, Enomoto Y, Takano M, Morita K, Kadota $\mathrm{E}$, et al. Genomic gains and losses in malignant mesothelioma demonstrated by FISH analysis of paraffin-embedded tissues. J Clin Pathol. 2012;65:77-82.

36. Testa JR, Cheung M, Pei J, Below JE, Tan Y, Sementino E, et al. Germline BAP1 mutations predispose to malignant mesothelioma. Nat Genet. 2011;43:1022-5.

37. Sheffield BS, Lorette J, Shen Y, Marra MA, Churg A. Immunohistochemistry for NF2, LATS1/2, and YAP/TAZ fails to separate benign from malignant mesothelial proliferations. Arch Pathol Lab Med. 2016;140:391.

38. Sekido Y. Inactivation of Merlin in malignant mesothelioma cells and the Hippo signaling cascade dysregulation. Pathol Int. 2011;61:331-44.

39. Thurneysen C, Opitz I, Kurtz S, Weder W, Stahel RA, FelleyBosco E. Functional inactivation of NF2/merlin in human mesothelioma. Lung Cancer. 2009;64:140-7.

40. Fleury-Feith J, Lecomte C, Renier A, Matrat M, Kheuang L, Abramowski $\mathrm{V}$, et al. Hemizygosity of $\mathrm{Nf} 2$ is associated with increased susceptibility to asbestos-induced peritoneal tumours. Oncogene. 2003;22:3799-805.

41. Meerang M, Bérard K, Friess M, Bitanihirwe BKY, Soltermann A, Vrugt B, et al. Low Merlin expression and high Survivin labeling index are indicators for poor prognosis in patients with malignant pleural mesothelioma. Mol Oncol. 2016;10:1255-65.

42. Altomare DA, Vaslet CA, Skele KL, De Rienzo A, Devarajan K, Jhanwar SC, et al. A mouse model recapitulating molecular features of human mesothelioma. Cancer Res. 2005;65:8090-5.

43. Cheng JQ, Lee WC, Klein MA, Cheng GZ, Jhanwar SC, Testa JR. Frequent mutations of NF2 and allelic loss from chromosome band 22q12 in malignant mesothelioma: evidence for a two-hit mechanism of NF2 inactivation. Genes Chromosomes Cancer. 1999;24:238-42.

44. Deguen B, Goutebroze L, Giovannini M, Boisson C, Van der Neut R, Jaurand MC, et al. Heterogeneity of mesothelioma cell lines as defined by altered genomic structure and expression of the NF2 gene. Int J Cancer. 1998;77:554-60. 